\title{
Predictors of Length of Hospital Stay, Mortality, and Outcomes Among Hospitalised COVID-19 Patients in Saudi Arabia: A Cross-Sectional Study
}

This article was published in the following Dove Press journal: Journal of Multidisciplinary Healthcare

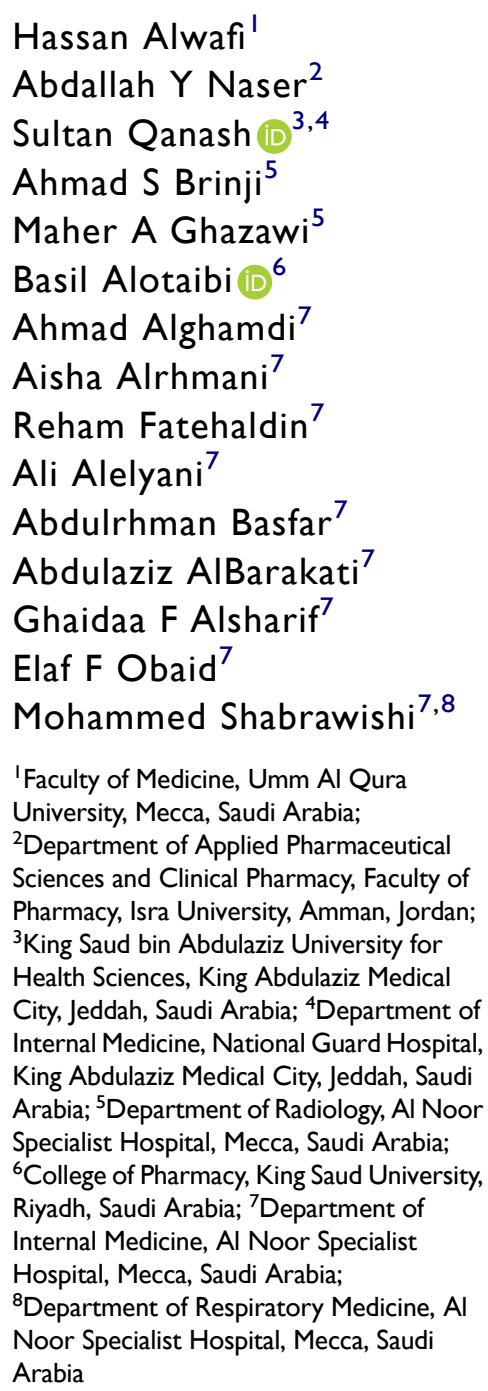

Correspondence: Mohammed Shabrawishi

Department of Respiratory Medicine, Al Noor Specialist Hospital, Mecca, 24242. Saudi Arabia

Tel +966555500592

Email shabrawishi.m@gmail.com
Background: COVID-19 pandemic is a major strain on health and economic systems, with rapidly increasing demand for in patients' facilities. Disease diagnosis and estimating patients at higher risk is important for the optimal management during the pandemic. This study aimed to identify the predictors of mortality and length of hospital stay in COVID-19 patients.

Methods: A retrospective cross-sectional study was conducted between March 2020 and August 2020 at Al-Noor Specialist Hospital in Mecca, Saudi Arabia. All patients who were admitted and had a confirmed COVID-19 diagnosis by a real-time polymerase chain reaction (PCR) were included in the study. Descriptive statistics were used to describe patients' demographic characteristics, laboratory findings, and clinical outcomes. Multiple logistic/linear regression analysis was used to identify predictors of death and length of stay at the hospital.

Results: A total of 706 patients were hospitalised for COVID-19. The mean age was 48.0 years (SD: 15.6 years). More than half of the patients $(68.5 \% ; n=292)$ were males. The median duration of stay at the hospital was 6.0 days (IQR: 300-10:00). The prevalence rate of venous thromboembolism (VTE) among the patients was $3.0 \%(\mathrm{n}=21)$. In the multivariate logistic regression analysis, age (AOR: 1.05; 1.02-1.09), patients with end-stage renal disease (AOR: 6.44; 2.20-18.87), low Oxygen saturation SPO2 (AOR: 9.92; 4.19-23.50), D.dimer $>0.5$ (AOR: 13.31; 5.45-32.49), ESR $>10 \mathrm{~mm} / \mathrm{h} \quad$ (AOR: 4.08; 1.72-9.68), Ferritin $>400 \mathrm{mcg} / \mathrm{L}$ (AOR: 18.55; 6.89-49.96), and Procalcitonin $>0.5 \mathrm{ug} / \mathrm{L} \quad(\mathrm{AOR}: 8.23$; 1.81-37.40) were associated with a higher risk of death among patients with COVID-19. Patients with VTE (AOR: 12.86; 3.07- 53.92) were at higher risk of death due to COVID-19. Conclusion: Hospitalised COVID-19 patients have multiple negative consequences in terms of their laboratory findings, signs and symptoms. Age and end-stage renal diseases have a significant impact on the mortality rate and the length of hospital stay among COVID-19 patients.

Keywords: COVID-19, hospitalisation, length of stay, survival, ICU, Saudi Arabia

\section{Introduction}

The severe acute respiratory syndrome coronavirus 2 (SARS-CoV-2), which was discovered in China in 2019, is an ongoing pandemic. ${ }^{1}$ In March 2021, it was reported that there are more than 117 million confirmed cases in the world, and the total number of deaths in the world is around 2,600,000 in 220 countries, with a mortality rate of around $2 \% .^{2}$ In Saudi Arabia, there were around 380,000 confirmed infected cases by January 2021, and a mortality rate of around $1.7 \% .^{3}$ 
Patients with COVID-19 usually complain of fever, cough, fatigue, anorexia, myalgia, and diarrhoea, ${ }^{4}$ but in severe illness, usually dyspnoea is the most common symptom often accompanied by hypoxemia. ${ }^{4}$ Mortality rates depend on patients who have severe respiratory failure related to interstitial pneumonia and acute respiratory distress syndrome, ${ }^{5}$ but higher mortality is found in association with older age, male sex, pre-existing cardiovascular diseases, uncontrolled diabetes, hypertension, asthma, chronic lung disease, and d-dimer greater than $1 \mu \mathrm{g} / \mathrm{mL}$ at admission. ${ }^{6}$ Length of hospital stay due to the COVID-19 depends on patients' clinical situation, however, it also depends on local guidelines in the institution or local health authority and the capacity of hospitals. ${ }^{7,8}$

COVID-19 pandemic is a major strain on health and economic systems, and the demand for inpatients' facilities is increasing with the increase in the number of infected cases. ${ }^{9}$ Predicting factors associated with the need for hospitalisation and length of stay can be important to help in aid prioritizing patients, decision-making and contingency planning. ${ }^{10}$ This study aimed to identify the predictors of mortality and length hospital of stay in COVID-19 patients.

\section{Methods}

\section{Study Design and Participants}

A retrospective cross-sectional study was conducted at AlNoor Specialist Hospital in Mecca, Saudi Arabia. Al-Noor Specialist Hospital is a tertiary hospital in Mecca, Saudi Arabia, and it is part of the Ministry of Health. The description of the study settings and the hospital has been described previously. ${ }^{11}$ All patients had a confirmed COVID-19 diagnosis by a real-time polymerase chain reaction (PCR). The PCR samples were obtained through a nasopharyngeal swap. All patients were admitted between March 15, 2020, and June 15, 2020 and they were followed up for a time to assess the clinical outcome; and the final date of follow-up was August 15, 2020. Data collection were between March 2020, and August 2020. All patients who were admitted and had a confirmed diagnosis of COVID-19 during the study period were included in the study.

\section{Data Collection and Study Variables}

Data were collected from patients' files and electronic records using a unique medical record number (MRN) for each patient. All data were collected, reviewed and checked by a medical team, including medical residents and a consultant pulmonologist. Data included the patient's demographics, clinical symptoms, comorbidity, and laboratory findings. Data were collected at the time of admission to the hospital. Patients were classified according to their severity based on the following category: mild, moderate, severe and, critically severe disease. The definition of these categories has been described previously. ${ }^{11}$

\section{Outcomes}

The primary outcome was predictors of patients' admission to an intensive care unit. Secondary outcomes were to identify predictors of length of hospital stay and mortality.

\section{Ethical Approval and Consent-to- Participate}

The study protocol and study methodology were approved by the Ministry of Health's Institutional Review Board (IRB), as well as the hospital (No H-02-K-076-0920386). Patients informed consent were obtained and patients were informed that their clinical data would be used for clinical or research purposes, while keeping all their personal information confidential. The ethical principles of the Declaration of Helsinki were adhered to during collection, handling, and storage of data, and all care was taken to protect patient confidentiality.

\section{Statistical Analysis}

Descriptive statistics were used to describe patients' demographics, laboratory findings, and clinical outcomes. Independent sample $t$-test was used to compare the mean value for continuous variables. A Chi-squared test/Fisher test was used to compare proportions for categorical variables. Multiple logistic/linear regression analysis was used to identify predictors of death and length of stay at the hospital, and a confidence interval of $95 \%(\mathrm{p}<0.05)$ was applied to represent the statistical significance of the results. All statistical analyses were conducted using SPSS (Statistical Package for the Social Sciences) version 25.0 software (SPSS Inc.).

\section{Results}

\section{Patients' Clinical Characteristics}

Table 1 below shows the characteristics of COVID-19 patients at presentation to the hospital. A total of 706 patients were hospitalised for COVID-19. The mean age was 48.0 years (SD: 15.6 years). More than half of the patients $(68.5 \% ; n=292)$ were males. The majority of 


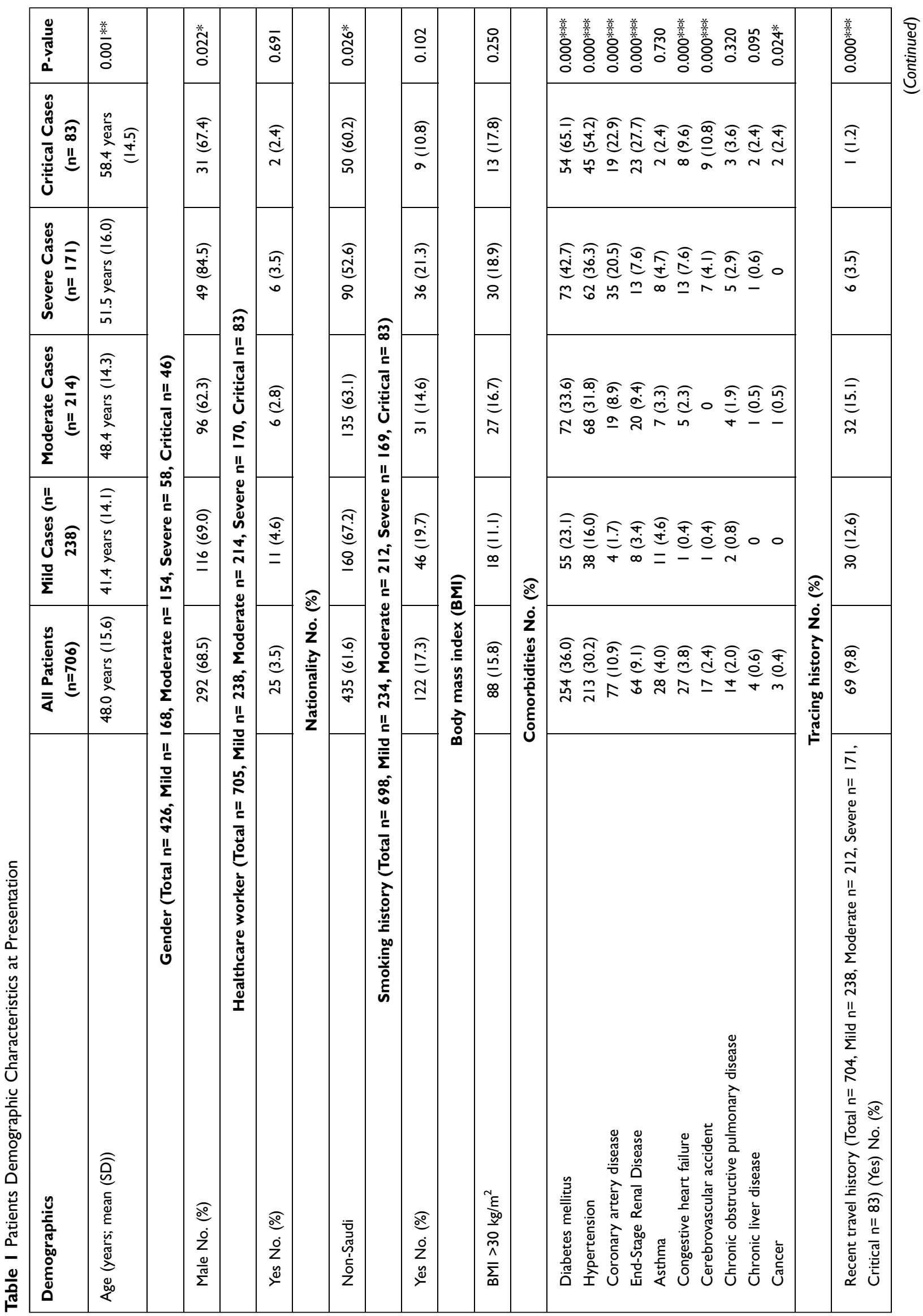




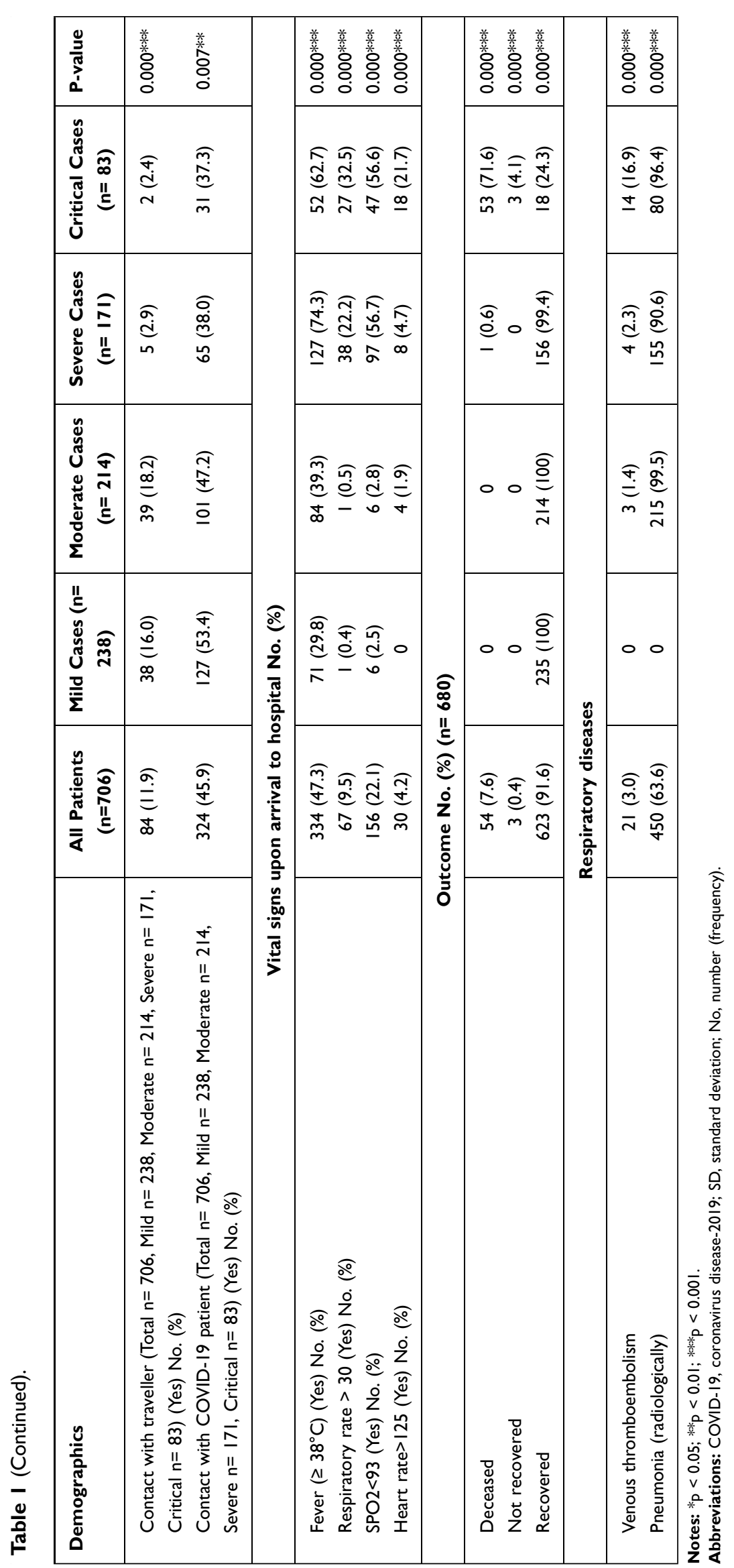


them were having mild to moderate cases. Twenty-five patients $(3.5 \%)$ reported working in the healthcare sector. More than half of them $(61.9 \% ; n=435)$ were non-Saudi. Around $17.4 \%(n=122)$ reported a history of smoking. The most common comorbidities were diabetes mellitus (DM) $(36.0 \%, n=254)$, hypertension $(30.2 \%, n=213)$, and coronary heart diseases $(10.9 \% ; n=77)$. Around $9.8 \%$ of the patients $(n=69)$ reported a recent travel history. Regarding the severity of the patients' case, $33.7 \%$ were mild, $30.3 \%$ were moderate, and $24.2 \%$ were severe, and $11.8 \%$ were critical and required intensive care unit (ICU) care. Regarding patients' vital signs upon arrival to hospital, $47.3 \%(\mathrm{n}=334)$ had fever (body temperature $>38^{\circ} \mathrm{C}$ ), $9.5 \%(n=67)$ had respiratory rate (RR) more than 30 , $22.1 \%(\mathrm{n}=156)$ had SPO2 < 93 , and $4.2 \%(\mathrm{n}=30)$ had heart rate $(\mathrm{HR})>125$.

Fever was the most common symptom at presentation $(72.4 \%, \mathrm{n}=511)$, followed by cough $(63.0 \%, \mathrm{n}=445)$, and shortness of breath $(56.4 \%, n=398)$ (Table 2). Fever, cough, shortness of breath, nausea/vomiting, headache, loss of taste and smell, sputum were more common across severe and critical cases compared to others.

\section{Laboratory Findings}

Around $16.5 \%(\mathrm{n}=116)$ of the patients had white blood cell $(\mathrm{WBC})>10,000,13.9 \%(\mathrm{n}=98)$ had $\mathrm{WBC}<4000$, and $21.0 \%(\mathrm{n}=148)$ of them had lymphocyte count $<1500$. About 12.6\% (89) had platelet count $<150$ and $29.9 \%$ ( $n=$ 210) had D.dimer $>0.5$. The proportion of patients who had WBC $>10,000$, lymphocyte count $<1500$, platelet count $<150$, and D.dimer $>0.5$ increase as the severity of the disease increase in a statistically significant pattern $(p<0.001)$. The mean Neutrophil-lymphocyte ratio (NLR) value was 9.9 (SD:33.5). The most common blood groups of COVID-19 patients were $\mathrm{A}+, \mathrm{O}+$, and $\mathrm{B}+$ accounting for $35.7 \%, 28.6 \%$, and $20.5 \%$ respectively (Table 3 ).

Regarding patients' inflammatory measures, around half of the patients had erythrocyte sedimentation rate (ESR) $>10 \mathrm{~mm} / \mathrm{h}$ and C-reactive protein (CRP) $>0.3 \mathrm{mg} /$ dl. One-third of the patients had Ferritin $>400 \mathrm{mcg} / \mathrm{L}$, and $1.8 \%$ of them had Procalcitonin $>0.5 \mathrm{ug} / \mathrm{L}$. Concerning patients' liver function tests, around one-third of the patients had AST $>40$ and ALT $>40$. Additionally, 40.1\% of them had lactate dehydrogenase $(\mathrm{LDH})>230 \mathrm{U} / \mathrm{L}$, and $6.2 \%$ had Bilirubin $>18.7$ umol/L. Regarding patients

Table 2 Patient Signs and Symptoms Stratified by Severity

\begin{tabular}{|c|c|c|c|c|c|c|}
\hline Variable & $\begin{array}{l}\text { All Patients } \\
\qquad(n=706)\end{array}$ & $\begin{array}{l}\text { Mild Cases } \\
(n=238)\end{array}$ & $\begin{array}{l}\text { Moderate Cases } \\
\qquad(n=2 \mid 4)\end{array}$ & $\begin{array}{c}\text { Severe Cases } \\
\qquad(n=|7|)\end{array}$ & $\begin{array}{l}\text { Critical Cases } \\
\qquad(n=83)\end{array}$ & P-value \\
\hline Fever & $511(72.4)$ & $143(60.1)$ & I52 (7I.0) & $150(87.7)$ & $66(79.5)$ & $0.000 * * *$ \\
\hline Cough & $445(63.0)$ & I 40 (58.8) & $128(59.8)$ & $117(68.4)$ & $60(72.3)$ & $0.047^{*}$ \\
\hline Shortness of breath & $398(56.4)$ & $90(37.8)$ & $97(45.3)$ & I43 (83.6) & $68(81.9)$ & $0.000 * * *$ \\
\hline Fatigue & $176(25.0)$ & $52(21.8)$ & $43(20.1)$ & $61(35.9)$ & $20(24.1)$ & $0.002^{* *}$ \\
\hline Nausea/vomiting & 118 (16.7) & $38(16.0)$ & $23(10.7)$ & $43(25.1)$ & 14 (16.9) & $0.003^{* *}$ \\
\hline Sore throat & $115(16.3)$ & $54(22.7)$ & $29(13.6)$ & $25(14.6)$ & $7(8.5)$ & $0.006 * *$ \\
\hline Myalgia & $108(15.3)$ & $31(13.0)$ & $24(11.3)$ & $42(24.6)$ & II (13.3) & $0.002^{* *}$ \\
\hline Headache & $102(14.4)$ & $31(13.0)$ & $28(13.1)$ & $31(18.1)$ & $12(14.5)$ & 0.460 \\
\hline Loss of taste & $98(13.9)$ & $30(12.6)$ & $21(9.8)$ & $21(12.3)$ & $26(31.3)$ & $0.000 * * *$ \\
\hline Loss of smell & $90(12.7)$ & $28(11.8)$ & $19(8.9)$ & $18(10.5)$ & $25(30.1)$ & $0.000 * * *$ \\
\hline Diarrhea & $52(7.4)$ & $19(8.0)$ & $13(6.1)$ & I5 (8.8) & $5(6.0)$ & 0.705 \\
\hline Sputum & $35(5.0)$ & $10(4.2)$ & $8(3.7)$ & II (6.4) & $6(7.2)$ & 0.447 \\
\hline Runny nose & $25(3.5)$ & $16(6.7)$ & $5(2.3)$ & $2(1.2)$ & $2(2.4)$ & $0.012^{*}$ \\
\hline Haemoptysis & $2(0.3)$ & 0 & 0 & I $(0.6)$ & I (I.2) & 0.228 \\
\hline
\end{tabular}

Notes: $*_{p}<0.05 ; * * p<0.01 ; * * * p<0.000$. 
Table 3 Laboratory Findings of the Study Participants Stratified by Severity

\begin{tabular}{|c|c|c|c|c|c|c|}
\hline Variable & $\begin{array}{l}\text { All } \\
\text { Patients } \\
(n=706)\end{array}$ & $\begin{array}{l}\text { Mild } \\
\text { Cases } \\
(n=238)\end{array}$ & $\begin{array}{l}\text { Moderate } \\
\text { Cases }(n= \\
214)\end{array}$ & $\begin{array}{l}\text { Severe } \\
\text { Cases }(n= \\
\quad 17 I)\end{array}$ & $\begin{array}{l}\text { Critical } \\
\text { Cases }(n= \\
\text { 83) }\end{array}$ & P-value \\
\hline \multicolumn{7}{|c|}{ Complete Blood Count } \\
\hline $\begin{array}{l}\text { WBC }>10,000 \text { (Total } n=701 \text {, Mild } n=234, \text { Moderate } n=214 \text {, } \\
\text { Severe } n=171 \text {, Critical } n=82 \text { ) (Yes) No. }(\%)\end{array}$ & $116(16.5)$ & $17(7.3)$ & $22(10.3)$ & 32 (18.7) & $45(54.9)$ & $0.000 * * *$ \\
\hline $\begin{array}{l}\text { WBC }<4000 \text { (Total } n=705, \text { Mild } n=238, \text { Moderate } n=214 \text {, } \\
\text { Severe } n=17 \mid \text {, Critical } n=82 \text { ) (Yes) No. }(\%)\end{array}$ & 98 (13.9) & $34(14.3)$ & 34 (I5.9) & $21(12.3)$ & $9(11.0)$ & 0.638 \\
\hline $\begin{array}{l}\text { Lymphocyte count }<1500 \text { (Total } n=704, \text { Mild } n=237 \\
\text { Moderate } n=214 \text {, Severe } n=17 \mid \text {, Critical } n=82) \text { (Yes) No. } \\
\text { (\%) }\end{array}$ & $148(2 \mid .0)$ & $25(10.5)$ & 40 (18.7) & $43(25.1)$ & $40(48.8)$ & $0.000 * * *$ \\
\hline NLR (Mean (SD)) & $9.9(33.5)$ & $\begin{array}{l}1.17 \\
(2.33)\end{array}$ & $1.23(2.28)$ & $9.2(66.9)$ & $12.8(18.8)$ & $0.000 * * *$ \\
\hline $\begin{array}{l}\text { Platelet }<150(\text { Total } n=704, \text { Mild } n=238, \text { Moderate } n=214 \text {, } \\
\text { Severe } n=170 \text {, Critical } n=82)(\text { Yes) No. }(\%)\end{array}$ & $89(12.6)$ & $21(8.8)$ & $23(10.7)$ & $22(12.9)$ & $23(28.0)$ & $0.000 * * *$ \\
\hline $\begin{array}{l}\text { D.dimer }>0.5 \text { (Total } n=703, \text { Mild } n=236, \text { Moderate } n=214 \text {, } \\
\text { Severe } n=171 \text {, Critical } n=82)(\text { Yes) No. }(\%)\end{array}$ & $210(29.9)$ & 27 (II.4) & $35(16.4)$ & 91 (53.2) & $57(69.5)$ & $0.000 * * *$ \\
\hline \multicolumn{7}{|c|}{ Blood Groups (Total $n=322$, Mild $n=82$, Moderate $n=86$, Severe $n=1 \mid 4$, Critical $n=40)($ Yes) No. $(\%)$} \\
\hline$A^{+}$ & 115 (35.7) & $34(4 \mid .5)$ & $21(24.4)$ & $44(38.6)$ & $16(40.0)$ & \multirow[t]{8}{*}{$0.000 * * *$} \\
\hline A- & $2(0.6)$ & 0 & 0 & $2(1.8)$ & 0 & \\
\hline$A B+$ & $22(6.8)$ & $5(6.1)$ & $5(5.8)$ & $9(7.9)$ & $3(7.5)$ & \\
\hline AB- & I (0.3) & $\mathrm{I}(\mathrm{I} .2)$ & 0 & 0 & 0 & \\
\hline$B+$ & $66(20.5)$ & $16(19.5)$ & 24 (27.9) & $20(17.5)$ & $6(15.0)$ & \\
\hline B- & $3(0.9)$ & I (I.2) & $2(2.3)$ & 0 & 0 & \\
\hline O+ & $92(28.6)$ & $19(23.2)$ & $27(3 \mid .4)$ & $32(28.1)$ & $14(35.0)$ & \\
\hline O- & $21(6.5)$ & $6(7.3)$ & $7(8.1)$ & $7(6.1)$ & I (2.5) & \\
\hline \multicolumn{7}{|c|}{ Inflammatory measures } \\
\hline $\begin{array}{l}\text { ESR }>10 \mathrm{~mm} / \mathrm{h}(\text { Total } n=704, \text { Mild } n=238, \text { Moderate } n=214 \text {, } \\
\text { Severe } n=170 \text {, Critical } n=82 \text { ) (Yes) No. }(\%)\end{array}$ & $366(52.0)$ & $67(28.2)$ & $99(46.3)$ & $139(81.8)$ & $61(74.4)$ & $0.000 * * *$ \\
\hline $\begin{array}{l}\text { CRP }>0.3 \mathrm{mg} / \mathrm{dl}(\text { Total } n=704, \text { Mild } n=238, \text { Moderate } n=213 \text {, } \\
\text { Severe } n=17 \mid \text {, Critical } n=82)(\text { Yes) No. }(\%)\end{array}$ & $340(48.3)$ & $65(27.3)$ & $97(45.6)$ & $132(77.2)$ & $46(56.1)$ & $0.000 * * *$ \\
\hline $\begin{array}{l}\text { Ferritin }>400 m c g / L \text { (Total } n=705, \text { Mild } n=238, \text { Moderate } n= \\
2 \mid 4 \text {, Severe } n=17 \mid \text {, Critical } n=82 \text { ) (Yes) No. }(\%)\end{array}$ & $204(28.9)$ & $40(16.8)$ & $43(20.1)$ & $76(44.4)$ & $45(54.9)$ & $0.000 * * *$ \\
\hline $\begin{array}{l}\text { Procalcitonin }>0.5 u g / L \text { (Total } n=705 \text {, Mild } n=237 \text {, Moderate } \\
n=216 \text {, Severe } n=170 \text {, Critical } n=82 \text { ) (Yes) No. }(\%)\end{array}$ & $13(1.8)$ & I (0.4) & $6(2.8)$ & $2(1.2)$ & $4(4.9)$ & $0.04 I^{*}$ \\
\hline \multicolumn{7}{|c|}{ Liver Function Tests (Total $n=705$, Mild $n=238$, Moderate $n=214$, Severe $n=171$, Critical $n=82)($ Yes) No. $(\%)$} \\
\hline AST $>40$ & $251(35.6)$ & $58(24.4)$ & $61(28.5)$ & $81(47.4)$ & $51(62.2)$ & $0.000 * * *$ \\
\hline ALT $>40$ & $237(33.6)$ & $55(23.1)$ & $56(26.2)$ & $81(47.4)$ & $45(54.9)$ & $0.000 * * *$ \\
\hline
\end{tabular}

(Continued) 
Table 3 (Continued).

\begin{tabular}{|c|c|c|c|c|c|c|}
\hline Variable & $\begin{array}{c}\text { All } \\
\text { Patients } \\
(n=706)\end{array}$ & $\begin{array}{c}\text { Mild } \\
\text { Cases } \\
(n=238)\end{array}$ & $\begin{array}{c}\text { Moderate } \\
\text { Cases (n= } \\
214)\end{array}$ & $\begin{array}{c}\text { Severe } \\
\text { Cases (n= } \\
\text { I7I) }\end{array}$ & $\begin{array}{c}\text { Critical } \\
\text { Cases (n= } \\
\text { 83) }\end{array}$ & P-value \\
\hline $\begin{array}{l}\mathrm{LDH}>230 \mathrm{U} / \mathrm{L} \text { (Total } n=704, \text { Mild } n=238, \text { Moderate } n=213 \text {, } \\
\text { Severe } n=171 \text {, Critical } n=82 \text { ) (Yes) No. }(\%)\end{array}$ & $282(40.1)$ & $51(21.4)$ & $62(29.1)$ & $108(63.2)$ & $61(74.4)$ & $0.000 * * *$ \\
\hline Bilirubin $>$ I8.7 umol/L & $44(6.2)$ & $6(2.5)$ & $9(4.2)$ & II (6.4) & $18(22.0)$ & $0.000 * * *$ \\
\hline \multicolumn{7}{|c|}{ Renal function tests (Total $n=705$, Mild $n=238$, Moderate $n=214$, Severe $n=17 \mid$, Critical $n=82)($ Yes) No. $(\%)$} \\
\hline Creatinine $>|| 5 \mathrm{umol} / \mathrm{L}$ & $121(17.2)$ & $13(5.5)$ & $28(13.1)$ & 34 (19.9) & $46(56.1)$ & $0.000 * * *$ \\
\hline $\begin{array}{l}\text { Urea }>6.04 \mathrm{mmol} / \mathrm{L} \text { (Total } \mathrm{n}=704, \text { Mild } n=237, \text { Moderate } n= \\
2 \mid 4, \text { Severe } n=17 \mid \text {, Critical } n=82 \text { ) (Yes) No. }(\%)\end{array}$ & |7| (24.3) & $19(8.0)$ & $46(21.5)$ & $57(33.3)$ & $49(59.8)$ & $0.000 * * *$ \\
\hline
\end{tabular}

Notes: *p < 0.05; ***p < 0.00I. Reference values; WBC: 4000-1000; lymphocyte: 1500-4000; NLR: 0.78-3.53; platelet: I50-400; D.dimer: 0-0.55; ESR: 0-10; CRP: 0-0.3; ferritin: 10-29I; procalcitonin: 0-0.5; AST: 10-34; ALT: 46-120; LDH: 80-230; bilirubin: 0-18.7; creatinine: 44-90; urea: 3.2-8.2.

Abbreviations: AST, aspartate transaminase; ALT, alanine transaminase; CRP, C-reactive protein; ESR, erythrocyte sedimentation rate; NLR, neutrophil-lymphocyte ratio; $\mathrm{LDH}$, lactate dehydrogenase; WBC, white blood cell.

kidney function tests, $17.2 \%$ of them had Creatinine $>115$ umol/L, and $24.3 \%$ had Urea $>6.04 \mathrm{mmol} / \mathrm{L}$ (Table 3).

\section{Factors Associated with Death}

The median duration of stay at the hospital was 6.0 days (IQR: $300-10: 00$ ). The duration of stay in the hospital ranged from one day to 55 days. The prevalence rate of venous thromboembolism (VTE) among the patients was $3.0 \%(n=21)$. More than half of the patients $(63.6 \%$; $n=$ 450) pneumonia radiologically. At the end of the followup period, a total of 623 patients $(91.6 \%)$ recovered. Three patients $(0.4 \%)$ did not recover at the end of the follow-up, and $7.6 \%$ of the patients $(n=54)$ deceased while the remaining either transferred to other facility or still in the hospital, at last, follow up. The severity of the cases affected the recovery rate and mortality rate in a statistically significant way $(\mathrm{p}>0.001)$ (Table 1$)$.

In the multivariate logistic regression analysis, the following risk factors were associated with a higher risk of death among patients with COVID-19. Age (AOR: $1.05 ; 1.02-1.09$ ), high respiratory rate (RR) (AOR: 153.90; 9.80-2416.60), low Oxygen saturation SPO2 (AOR: 9.92; 4.19-23.50), D.dimer $>0.5$ (AOR: 13.31; 5.45-32.49), ESR $>10 \mathrm{~mm} / \mathrm{h} \quad(A O R: \quad 4.08 ; 1.72-9.68)$, Ferritin $>400 \mathrm{mcg} / \mathrm{L} \quad$ (AOR: 18.55; 6.89-49.96), and Procalcitonin $>0.5 \mathrm{ug} / \mathrm{L}$ (AOR: 8.23; 1.81-37.40). Patients with VTE (AOR: 12.86; 3.07-53.92) were at higher risk of death due to COVID-19. End-stage renal diseases were identified to increase the risk of COVID-19. For further details, please refer to Table 4 .

\section{Factors Associated with Increased Length of Stay}

Several factors were associated with increased risk of the length of stay, including comorbidities such as congestive heart failure, cerebrovascular accident, and COPD, fever, D.dimer value of more than $>0.5$, $\mathrm{WBC}>10,000$, $\mathrm{ESR}>10 \mathrm{~mm} / \mathrm{h}, \mathrm{CRP}>0.3 \mathrm{mg} / \mathrm{dl}$, ferritin $>400 \mathrm{mcg} / \mathrm{L}$, procalcitonin $>0.5 \mathrm{ug} / \mathrm{L}, \mathrm{LDH}>230 \mathrm{U} / \mathrm{L}$, creatinine $>115$ umol $/ \mathrm{L}$, and blood group $\mathrm{O}(\mathrm{p}<0.05)$, for further details, please refer to Table 5 .

\section{Discussion}

In this cross-sectional study, we investigated the predictors of mortality and length of stay in hospital among hospitalised patients with COVID-19. The key findings of this study are that age, patients with chronic comorbidities, patients with VTE and radiological evidence of pneumonia, and higher D-dimer values were important risk factors that were associated with a higher risk of death and increased length of stay in hospital.

Our findings revealed the significant impact of age and chronic conditions on the mortality rate and the length of hospital stay. It comes as no surprise that the escalated rate of mortality and prolonged hospital stay was associated with older patients. Thus this study substantiates the previous findings of the literature. ${ }^{12}$ This may also be due to their weak immune system and some behavioural responses in the measures taken. ${ }^{13,14}$ Chronic diseases demonstrate a significant influence on the outcomes; diabetes patients were more vulnerable to fatal consequences 
Table 4 Logistic Regression to Identify Risk Factors of Death

\begin{tabular}{|c|c|c|c|c|}
\hline Demographics & Odds Ratio for Death ${ }^{a}$ & $95 \% \mathrm{Cl}$ & Odds Ratio for Death ${ }^{\mathrm{b}}$ & $95 \% \mathrm{Cl}$ \\
\hline Age & 1.06 & $(1.04-1.08)^{* * *}$ & 1.05 & $(1.02-1.09)^{* *}$ \\
\hline \multicolumn{5}{|c|}{ Gender } \\
\hline Female (Reference category) & \multicolumn{2}{|c|}{1.00} & \multicolumn{2}{|c|}{1.00} \\
\hline Male & 0.77 & $(0.39-1.54)$ & 0.30 & $(0.1 \mathrm{I}-0.8 \mathrm{I})^{*}$ \\
\hline \multicolumn{5}{|c|}{ Smoking history } \\
\hline No (Reference category) & \multicolumn{2}{|c|}{1.00} & \multicolumn{2}{|c|}{1.00} \\
\hline Yes & 0.36 & $(0.13-1.02)$ & 0.49 & $(0.13-1.91)$ \\
\hline \multicolumn{5}{|c|}{ BMI } \\
\hline BMI $<30 \mathrm{~kg} / \mathrm{m}^{2}$ (Reference category) & \multicolumn{2}{|l|}{1.00} & \multicolumn{2}{|c|}{1.00} \\
\hline $\mathrm{BMI}>30 \mathrm{~kg} / \mathrm{m}^{2}$ & 1.24 & $(0.59-2.63)$ & 0.25 & $(0.06-1.01)$ \\
\hline \multicolumn{5}{|c|}{ Comorbidities (not having the disease is the reference category) } \\
\hline Diabetes mellitus & 4.82 & $(2.63-8.85)^{* * *}$ & 1.64 & $(0.59-4.54)$ \\
\hline Hypertension & 5.91 & $(3.24-10.77)^{* * *}$ & 1.63 & $(0.55-4.85)$ \\
\hline Coronary artery disease & 3.04 & $(1.54-5.99)^{* *}$ & 1.08 & $(0.28-4.17)$ \\
\hline End-Stage Renal Disease & 6.97 & $(3.69-13.17)^{* * *}$ & 6.44 & $(2.20-18.87)^{* *}$ \\
\hline Asthma & 0.42 & $(0.06-3.13)$ & 0.80 & $(0.07-8.82)$ \\
\hline Congestive heart failure & 3.59 & $(1.38-9.32)^{* *}$ & 0.85 & $(0.17-4.31)$ \\
\hline Cerebrovascular accident & 10.18 & $(3.63-28.54)^{* * *}$ & 2.09 & $(0.37-11.92)$ \\
\hline Chronic obstructive pulmonary disease & 0.89 & $(0.11-6.90)$ & \multicolumn{2}{|l|}{-} \\
\hline Chronic liver disease & 11.92 & $(1.65-86.38)^{*}$ & \multicolumn{2}{|l|}{-} \\
\hline Cancer & 23.96 & $(2.14-268.68)^{*}$ & \multicolumn{2}{|l|}{-} \\
\hline \multicolumn{5}{|c|}{ Vital signs upon arrival to hospital (having the normal range is the reference category) } \\
\hline Fever $\left(\geq 38^{\circ} \mathrm{C}\right)($ Yes$)$ & 1.28 & $(0.73-2.24)$ & 1.46 & $(0.68-3.13)$ \\
\hline Respiratory rate > 30 (Yes) & 7.09 & $(3.66-13.73)^{* * *}$ & 153.90 & $(9.80-2416.60)^{* * *}$ \\
\hline SPO2<93 (Yes) & 2.63 & $(1.46-4.73)^{* *}$ & 9.92 & $(4.19-23.50)^{* * *}$ \\
\hline Heart rate> 125 (Yes) & 12.87 & $(5.74-28.86)^{* * *}$ & 19.82 & $(5.22-75.25)^{* * *}$ \\
\hline \multicolumn{5}{|c|}{ Outcome } \\
\hline Venous thromboembolism (Yes) & 17.54 & $(6.90-44.6 I)^{* * *}$ & 12.86 & $(3.07-53.92)^{* * *}$ \\
\hline Pneumonia Radiologically(Yes) & 2.35 & $(1.19-4.61)^{*}$ & 1.37 & $(0.95-1.98)$ \\
\hline \multicolumn{5}{|c|}{ Complete Blood Count (having the normal range is the reference category) } \\
\hline WBC >10,000 (Yes) & 12.36 & $(6.74-22.68)^{* * *}$ & 16.47 & $(6.78-40.00)^{* * *}$ \\
\hline WBC $<4000$ (Yes) & 0.34 & $(0.10-1.11)$ & 0.31 & $(0.07-1.48)$ \\
\hline Lymphocyte count $<1500$ (Yes) & 5.70 & $(3.19-10.17)^{* * *}$ & 10.17 & $(4.29-24.14)^{* * *}$ \\
\hline NLR (Mean (SD)) & 1.00 & $(1.00-1.01)$ & 1.23 & $(1.15-1.32)^{* * *}$ \\
\hline
\end{tabular}


Table 4 (Continued).

\begin{tabular}{|c|c|c|c|c|}
\hline Demographics & Odds Ratio for Death ${ }^{\mathrm{a}}$ & $95 \% \mathrm{Cl}$ & Odds Ratio for Death ${ }^{b}$ & $95 \% \mathrm{Cl}$ \\
\hline Platelet < I50 (Yes) & 5.73 & $(3.12-10.53)^{* * *}$ & 9.92 & $(4.01-24.52)^{* * *}$ \\
\hline D.dimer >0.5 (Yes) & 8.47 & $(4.48-16.01)^{* * *}$ & $|3.3|$ & $(5.45-32.49)^{* * *}$ \\
\hline \multicolumn{5}{|c|}{ Inflammatory measures (having the normal range is the reference category) } \\
\hline $\mathrm{ESR}>10 \mathrm{~mm} / \mathrm{h}(\mathrm{Yes})$ & 2.89 & $(1.54-5.42)^{* *}$ & 4.08 & $(1.72-9.68)^{* *}$ \\
\hline CRP>0.3 mg/dl (Yes) & 0.90 & $(0.52-1.55)$ & 0.84 & $(0.39-1.80)$ \\
\hline Ferritin $>400 \mathrm{mcg} / \mathrm{L}$ (Yes) & 3.89 & $(2.19-6.91)^{* * *}$ & 18.55 & $(6.89-49.96)^{* * *}$ \\
\hline Procalcitonin $>0.5 \mathrm{ug} / \mathrm{L}$ (Yes) & 5.58 & $(1.66-18.77)^{* *}$ & 8.23 & $(1.81-37.40)^{* *}$ \\
\hline \multicolumn{5}{|c|}{ Liver Function Tests (having the normal range is the reference category) } \\
\hline AST $>40$ (Yes) & 3.71 & $(2.07-6.67)^{* * *}$ & 8.85 & $(3.64-21.50)^{* * *}$ \\
\hline ALT $>40$ (Yes) & 2.41 & $(1.37-4.24)^{* *}$ & 4.78 & $(2.11-10.83)^{* * *}$ \\
\hline LDH>230 U/L (Yes) & 6.09 & $(3.13-11.82)^{* * *}$ & 21.26 & $(7.61-59.40)^{* * *}$ \\
\hline Bilirubin $>18.7$ umol/L (Yes) & 1.51 & $(1.14-2.00)^{* *}$ & 15.06 & $(4.63-48.99)^{* * *}$ \\
\hline \multicolumn{5}{|c|}{ Renal function tests (having the normal range is the reference category) } \\
\hline Creatinine $>$ II 15 umol/L (Yes) & 18.00 & $(9.46-34.25)^{* * *}$ & 36.33 & $(13.65-96.66)^{* * *}$ \\
\hline Urea>6.04 mmol/L (Yes) & 4.48 & $(2.54-7.90)^{* * *}$ & 15.27 & $(6.29-37.07)^{* * *}$ \\
\hline \multicolumn{5}{|c|}{ Blood Groups (blood group A is the reference category) } \\
\hline B & 0.35 & $(0.08-1.47)$ & 0.51 & $(0.11-2.36)$ \\
\hline$A B$ & - & - & 1.08 & $(0.02-54.95)$ \\
\hline O & 1.52 & $(0.77-2.99)$ & 1.50 & $(0.57-3.96)$ \\
\hline
\end{tabular}

Notes: $*_{\mathrm{p}}<0.05$; ** $<0.01$; *** $<0.00 \mathrm{I}$. ${ }^{\mathrm{a} B i n a r y}$ logistic regression. ${ }^{\mathrm{b}}$ Multiple logistic regression adjusted for the following variables (age, gender, and comorbidities). Reference values; WBC: 4000-1000; lymphocyte: 1500-4000; NLR: 0.78-3.53; platelet: 150-400; D.dimer: 0-0.55; ESR: 0-10; CRP: 0-0.3; ferritin: 10-291; procalcitonin: 0-0.5; AST: 10-34; ALT: 46-I20; LDH: 80-230; bilirubin: 0-18.7; creatinine: 44-90; urea: 3.2-8.2.

Abbreviations: AST, aspartate transaminase; ALT, alanine transaminase; CRP, C-reactive protein; ESR, erythrocyte sedimentation rate; NLR, neutrophil-lymphocyte ratio; LDH, lactate dehydrogenase; WBC, white blood cell.

and longer hospitalisation compared to non-DM patients, which is consistent with results reported from the previous study. ${ }^{15}$ Hypertensive patients have a propensity to express high mortality rate and stay hospitalised longer. Likewise, pre-existent cardiovascular and cerebrovascular events carry a high risk of death and a longer duration of hospitalisation. The effect of SARS-COV-2 on the vascular endothelium could be explained by the current understanding that angiotensin-converting enzyme 2 (ACE2) cellular receptors as the entry sites of SARS-COV2 as shown in different studies. ${ }^{16,17}$

There are several explanations for these results, ReninAngiotensin system (RAAS) and inflammatory cytokines have been mentioned as mediators in severe outcomes among hypertensive patients. ${ }^{18}$ Furthermore, the frequent utilisation of angiotensin-converting enzyme inhibitors (ACEIs) can lead to a decrease in angiotensin-converting enzyme (ACE) and an increase in the expression of ACE2 in the lungs which eventually facilitates the invasion of Covid-19 virus, ${ }^{19,20}$ nonetheless this still controversial as illustrated by other studies. ${ }^{21}$ Further, the severe viral virulence may lead to high oxygen demand, physiological and reflex tachycardia and aggravates the manifestation of coronary artery diseases accompanied by respiratory distress, finally, unfavourable outcomes will present. ${ }^{22}$ Patients with chronic liver diseases (CLD) were also more susceptible to fatal consequences either death or a longer hospitalisation period. The confirmed laboratory findings emphasise the negative impact of Covid-19 on liver functions. The previous study elucidated the high mortality rate among CLD 
Table 5 Linear Regression to Identify Predictors of Length of Stay

\begin{tabular}{|c|c|c|c|c|c|c|}
\hline \multirow[t]{2}{*}{ Demographics } & \multicolumn{3}{|c|}{ Model a } & \multicolumn{3}{|c|}{ Model b } \\
\hline & B & SE & B & B & SE & B \\
\hline Age & 0.07 & 0.02 & $0.145^{* * *}$ & -0.013 & 0.02 & -0.03 \\
\hline \multicolumn{7}{|c|}{ Gender } \\
\hline Female & 0.93 & 0.68 & -0.07 & -1.21 & 0.66 & -0.09 \\
\hline \multicolumn{7}{|c|}{ Smoking history } \\
\hline Yes & 3.04 & $0.7 \mathrm{I}$ & $-0.16 * * *$ & -1.75 & 1.03 & -0.11 \\
\hline \multicolumn{7}{|c|}{ BMI } \\
\hline $\mathrm{BMI}>30 \mathrm{~kg} / \mathrm{m}^{2}$ & 0.12 & 0.77 & 0.01 & 4.27 & 6.70 & 0.04 \\
\hline \multicolumn{7}{|c|}{ Comorbidities (not having the disease is the reference category) } \\
\hline Diabetes mellitus & 2.46 & 0.56 & $0.17 * * *$ & 0.73 & 0.76 & 0.05 \\
\hline Hypertension & 3.07 & 0.58 & $0.20 * * *$ & 0.55 & 0.82 & 0.04 \\
\hline Coronary artery disease & 3.06 & 0.86 & $0.13 * * *$ & -1.00 & 1.21 & -0.04 \\
\hline End-Stage Renal Disease & 7.68 & 0.91 & $0.31 * * *$ & -1.04 & 2.00 & -0.03 \\
\hline Asthma & 1.20 & 1.40 & 0.03 & -0.28 & 2.16 & -0.01 \\
\hline Congestive heart faliure & 3.77 & 1.40 & $0.10 * *$ & -1.04 & 2.00 & -0.03 \\
\hline Cerebrovascular accident & 5.31 & 1.74 & $0.12 * *$ & 7.82 & 2.18 & $0.17^{* * *}$ \\
\hline Chronic obstructive pulmonary disease & 3.93 & 1.92 & $0.08^{*}$ & 3.63 & 3.96 & 0.05 \\
\hline Chronic liver disease & 3.08 & 3.59 & 0.03 & 2.76 & 4.83 & 0.03 \\
\hline Cancer & -0.60 & 4.14 & -0.01 & -4.88 & 3.98 & -0.06 \\
\hline \multicolumn{7}{|c|}{ Vital signs upon arrival to hospital } \\
\hline Fever $\left(\geq 38^{\circ} \mathrm{C}\right)($ Yes $)$ & 2.19 & 0.54 & $0.15^{* * *}$ & 1.83 & 0.64 & $0.13^{* *}$ \\
\hline Respiratory rate > 30 (Yes) & 5.15 & 0.90 & $0.21 * * *$ & 0.83 & 2.38 & 0.02 \\
\hline SPO2<93 (Yes) & 1.90 & 0.48 & $0.15^{* * *}$ & 0.21 & 0.97 & 0.01 \\
\hline Heart rate> 125 (Yes) & 4.95 & 1.32 & $0.14 * * *$ & 0.54 & 1.82 & 0.01 \\
\hline \multicolumn{7}{|c|}{ Outcome } \\
\hline Venous thromboembolism (Yes) & 5.04 & 1.62 & $0.12 * *$ & 2.19 & 2.06 & 0.05 \\
\hline Pneumonia (Yes) & 2.79 & 0.42 & $0.24 * * *$ & 1.19 & 0.44 & $0.13^{* *}$ \\
\hline \multicolumn{7}{|c|}{ Complete Blood Count } \\
\hline WBC >10,000 (Yes) & 4.02 & 0.72 & $0.21 * * *$ & 2.37 & 0.87 & $0.13^{* *}$ \\
\hline WBC < 4000 (Yes) & -0.38 & 0.79 & -0.02 & 1.69 & 0.93 & 0.09 \\
\hline Lymphocyte count <1500 (Yes) & 1.02 & 0.67 & 0.06 & 1.13 & 0.89 & 0.06 \\
\hline NLR (Mean (SD)) & 0.00 & 0.01 & 0.02 & 0.07 & 0.06 & 0.06 \\
\hline Platelet < I50 (Yes) & 0.24 & 0.81 & 0.01 & 0.45 & 1.07 & 0.02 \\
\hline
\end{tabular}


Table 5 (Continued).

\begin{tabular}{|c|c|c|c|c|c|c|}
\hline \multirow[t]{2}{*}{ Demographics } & \multicolumn{3}{|c|}{ Model a } & \multicolumn{3}{|c|}{ Model b } \\
\hline & B & SE & B & B & SE & B \\
\hline D.dimer >0.5 (Yes) & 3.57 & 0.58 & $0.23 * * *$ & 2.57 & 0.87 & $0.15^{* *}$ \\
\hline \multicolumn{7}{|c|}{ Inflammatory measures } \\
\hline ESR > 10 mm/h (Yes) & 2.09 & 0.54 & $0.15^{* * *}$ & $\mathrm{I} .64$ & 0.64 & $0.12^{*}$ \\
\hline CRP>0.3 mg/dl (Yes) & 2.10 & 0.54 & $0.15^{* * *}$ & 2.74 & 0.64 & $0.20 * * *$ \\
\hline Ferritin>400mcg/L (Yes) & 4.59 & 0.57 & $0.29 * * *$ & 3.70 & 0.72 & $0.24 * * *$ \\
\hline Procalcitonin $>0.5 \mathrm{ug} / \mathrm{L}$ (Yes) & 4.46 & 2.00 & $0.08^{*}$ & 4.94 & 1.78 & $0.13 * *$ \\
\hline \multicolumn{7}{|c|}{ Liver Function Tests } \\
\hline AST $>40$ & -0.27 & 0.57 & -0.02 & -0.81 & 0.69 & -0.06 \\
\hline ALT $>40$ & -0.12 & 0.57 & -0.01 & -0.82 & 0.71 & -0.06 \\
\hline LDH>230 U/L (Yes) & 2.09 & 0.55 & $0.14 * * *$ & 2.32 & 0.70 & $0.16 * *$ \\
\hline Bilirubin> $>8.7 \mathrm{umol} / \mathrm{L}$ & 0.61 & 0.43 & 0.05 & 1.03 & 1.48 & 0.04 \\
\hline \multicolumn{7}{|c|}{ Renal function tests } \\
\hline Creatinine $>|| 5 \mathrm{umol} / \mathrm{L}$ & 4.90 & 0.69 & $0.26 * * *$ & 2.44 & 1.09 & $0.13^{*}$ \\
\hline Urea>6.04 mmol/L (Yes) & 2.89 & 0.54 & $0.20 * * *$ & 1.69 & 0.91 & 0.10 \\
\hline \multicolumn{7}{|c|}{ Blood Groups } \\
\hline B & -1.38 & 0.92 & -0.06 & -1.03 & 1.12 & -0.04 \\
\hline$A B$ & -3.58 & 3.22 & -0.04 & 2.70 & 4.83 & 0.03 \\
\hline O & 0.52 & 0.74 & 0.03 & 3.43 & 0.95 & $0.17 * * *$ \\
\hline
\end{tabular}

Notes: ${ }^{*}<<0.05 ; *_{p}<0.01 ; *^{* * *} p<0.001$. Model a: Univariate linear regression. Model b: Multiple linear regression adjusted for the following variables (age, gender and comorbidities).

Abbreviations: AST, aspartate transaminase; ALT, alanine transaminase; CRP, C-reactive protein; ESR, erythrocyte sedimentation rate; NLR, neutrophil-lymphocyte ratio; $\mathrm{LDH}$, lactate dehydrogenase; WBC, white blood cell; B, the average change in the dependent variable associated with a I unit change in the independent variable, statistically controlling for the other independent variables; SE, it is the standard deviation of its sampling distribution or an estimate of that standard deviation; B, a statistical measure that compares the strength of the effect of each individual independent variable to the dependent variable.

patient who they are Covid-19 infected..$^{23,24}$ Our findings also highlighted the high mortality rate and the long period of hospitalisation among cancer patients. The nature of cancer and the antineoplastic agents compromise the immune system. Consequently, it spikes the probability of lethal and severe infection of the Covid-19 virus among these patients. Moreover, the redundant clinical visits for follow up and chemotherapy dose also expose the patients to the infection. ${ }^{25}$

The hypercoagulability of Covid-19 patients was observed and confirmed by laboratory findings. Venous thrombus embolism was one of the poor outcomes among covid-19 patients with a significant correlation and high odd ratios. The severe infection and long bed- ridden interval in ICU dysregulate the homeostasis of the cascade system by activating the inflammatory cytokines. ${ }^{26}$ Not withstanding, the pathogeneses of VTEinduced by Covid-19 are complex and multifactorial. Our study provides further evidence of Covid-19 pneumonia as a predictor for the high mortality rate and prolonged hospitalisation. These findings support the aggressive preventive measures that be taken to halt the mortality rate among these patients.

In our study, we found no significant difference between blood group type and the risk of death. However, we found a significant difference in the duration of hospital stay for patients with blood group O. Previous reports showed a reduced prevalence of Covid-19 
infection in blood group $\mathrm{O} .{ }^{27}$ However, this was later contradicted, as some other published report suggested that there is no link between Covid-19 infection and type of blood group. ${ }^{28,29}$ Future studies on a larger scale and different populations are needed to investigate this association.

In our study and similar to published reports, patients with chronic obstructive pulmonary disease (COPD) were found to be at higher risk for a severe outcome, ${ }^{30}$ likely due to the fact that these patients usually have reduced lung function along with various comorbidities. ${ }^{31}$ Interestingly, on the other hand, patients with pre-existent asthma did not have a risk of worse outcome, ${ }^{32}$ and this could be partly explained by the lower expression of ACE2 in asthmatic bronchial epithelium. ${ }^{33}$

Obesity is one of the major comorbidities to be considered. Above increasing the risk of different complications such as DM, liver diseases and cardiovascular diseases, we observed obesity also increases the mortality rate and the demand on intensive care facilities among SARS-COV-2 patients, which augments the findings in a previous systematic review. ${ }^{34}$ Henceforth, obesity is one of the potential predictors for the study outcomes. However, the underlying mechanism behind the bad prognosis of obese patients still unknown.

We believe that our results are similar to the literature, it may help in earlier risk stratification, and triage of COVID-19 patients admitted to the hospital and in reducing the overload on emergency departments visits and intensive care units in order to facilitate COVID-19 cases and other emergency care cases. Some factors identified in this study, such as older age and comorbidities, may help policymakers and guidelines in their recommendations about prioritising patients based on their symptoms and may help to improve the patient's care. Furthermore, the founded risk factors might be helpful in establishing a scoring system that can be applied to predict mortality and appropriate management plan.

This study has some limitations. First, the study population only included patients from a single-centre hospital in Saudi Arabia. Second, the cross-sectional study design restricted our ability to identify causality between study variables.

\section{Conclusion}

Hospitalised COVID-19 patients have multiple negative consequences in term of their laboratory findings, signs and symptoms. Age and chronic conditions have a significant impact on the mortality rate and the length of hospital stay among COVID-19 patients. Earlier risk stratification of the COVID-19 patients admitted to the hospital is recommended.

\section{Author Contributions}

Conceptualization, Hassan Alwafi, Mohammed Shabrawishi, Sultan Qanash, Abdallah Y Naser; Data curation, Mohammed Shabrawishi, Ahmad S Brinji, Maher A Ghazawi, Ahmad Alghamdi, Aisha Alrhmani, Reham Fatehaldin, Ali Alelyani, Abdulrhman Basfar, Abdulaziz Al Barakati, Ghaidaa F Alsharif, Elaf F Obaid; Formal analysis, Abdallah Y Naser, Hassan Alwafi; Investigation, Mohammed Shabrawishi, Sultan Qanash, Abdallah Y Naser, Ahmad S Brinji and Hassan Alwafi; Methodology, Mohammed Shabrawishi, Abdallah Y Naser, Sultan Qanash and Hassan Alwafi; Project administration, Mohammed Shabrawishi and Hassan Alwafi; Resources, Ahmad Alghamdi, Aisha Alrhmani, Reham Fatehaldin, Ali Alelyani, Abdulrhman Basfar, Abdulaziz Al Barakati, Ghaidaa F Alsharif, Elaf F Obaid; Supervision, Mohammed Shabrawishi and Hassan Alwafi; Validation, Mohammed Shabrawishi, Abdallah Y Naser, Maher A Ghazawi, Ahmad S Brinji and Hassan Alwafi; Writing - original draft, Mohammed Shabrawishi, Abdallah Y Naser, Sultan Qanash, Basil Alotaibi and Hassan Alwafi; Writing- review \& editing, Mohammed Shabrawishi, Sultan Qanash, Abdallah Y Naser, Ahmad S Brinji, Maher A Ghazawi, Ahmad Alghamdi, Aisha Alrhmani, Reham Fatehaldin, Ali Alelyani, Abdulrhman Basfar, Abdulaziz Al Barakati, Ghaidaa F Alsharif, Elaf F Obaid, Basil Alotaibi and Hassan Alwafi. All authors contributed to data analysis, drafting or revising the article, have agreed on the journal to which the article will be submitted, gave final approval of the version to be published, and agree to be accountable for all aspects of the work.

\section{Disclosure}

The authors have stated explicitly that there are no conflicts of interest in connection with this article.

\section{References}

1. Zhu N, Zhang D, Wang W, et al. A Novel Coronavirus from patients with Pneumonia in China, 2019. N Engl J Med. 2020;382(8):727-733. doi:10.1056/NEJMoa2001017

2. World Health Organization. Coronavirus disease 2019 (COVID-19) Situation Report-209; 2020. Available from: https://www.who.int/ emergencies/diseases/novel-coronavirus-2019/situation-reports. 
3. (SCDC) Scfdpac. COVID-19 disease daily report November 18 2020; 2020. Available from: https://covid19.cdc.gov.sa/dailyupdates. Accessed April 04, 2021.

4. World Health Organization. Coronavirus disease (COVID-19) outbreak 2020; 2020. Available from: https://www.who.int/emergencies/ diseases/novel-coronavirus-2019. Accessed April 04, 2021.

5. Ranieri VM, Rubenfeld GD, Thompson BT, et al. Acute respiratory distress syndrome: the Berlin Definition. JAMA. 2012;307 (23):2526-2533. doi:10.1001/jama.2012.5669

6. Zhou F, Yu T, Du R, et al. Clinical course and risk factors for mortality of adult inpatients with COVID-19 in Wuhan, China: a retrospective cohort study. Lancet (London, England). 2020;395 (10229):1054-1062. doi:10.1016/S0140-6736(20)30566-3

7. Wu Z, McGoogan JM. Characteristics of and important lessons from the Coronavirus disease 2019 (COVID-19) outbreak in China: summary of a report of 72314 cases from the Chinese Center for Disease Control and Prevention. JAMA. 2020;323(13):1239-1242. doi:10.1001/jama.2020.2648

8. Chen S, Zhang Z, Yang J, et al. Fangcang shelter hospitals: a novel concept for responding to public health emergencies. Lancet (London, England). 2020;395(10232):1305-1314. doi:10.1016/ S0140-6736(20)30744-3

9. Xing C, Zhang R. COVID-19 in China: responses, challenges and implications for the health system. Healthcare. 2021;9(1):82. doi:10.3390/healthcare9010082

10. Rees EM, Nightingale ES, Jafari Y, et al. COVID-19 length of hospital stay: a systematic review and data synthesis. BMC Med. 2020;18(1):270. doi:10.1186/s12916-020-01726-3

11. Shabrawishi M, Al-Gethamy MM, Naser AY, et al. Clinical, radiological and therapeutic characteristics of patients with COVID-19 in Saudi Arabia. PLoS One. 2020;15(8):e0237130. doi:10.1371/journal. pone. 0237130

12. Wei C, Liu Y, Liu Y, et al. Clinical characteristics and manifestations in older patients with COVID-19. BMC Geriatr. 2020;20(1):395. doi:10.1186/s12877-020-01811-5

13. Sun Z, Yang B, Zhang R, Cheng X. Influencing factors of understanding COVID-19 risks and coping behaviors among the elderly population. Int J Environ Res Public Health. 2020;17(16):5889. doi:10.3390/ijerph17165889

14. Sun Z, Cheng X, Zhang R, Yang B. Factors influencing rumour re-spreading in a public health crisis by the middle-aged and elderly populations. Int J Environ Res Public Health. 2020;17(18):6542. doi:10.3390/ijerph17186542

15. Alguwaihes AM, Al-Sofiani ME, Megdad M, et al. Diabetes and Covid-19 among hospitalized patients in Saudi Arabia: a single-centre retrospective study. Cardiovasc Diabetol. 2020;19 (1):205. doi:10.1186/s12933-020-01184-4

16. Kreutz R, Algharably EAE, Azizi M, et al. Hypertension, the renin-angiotensin system, and the risk of lower respiratory tract infections and lung injury: implications for COVID-19. Cardiovasc Res. 2020;116(10):1688-1699. doi:10.1093/cvr/cvaa097

17. Imai Y, Kuba K, Penninger JM. The discovery of angiotensin-converting enzyme 2 and its role in acute lung injury in mice. Exp Physiol. 2008;93(5):543-548. doi:10.1113/ expphysiol.2007.040048

18. Wang Y, Chen B, Li Y, et al. The use of renin-angiotensin-aldosterone system (RAAS) inhibitors is associated with a lower risk of mortality in hypertensive COVID-19 patients: a systematic review and meta-analysis. J Med Virol. 2021;93(3):1370-1377. doi:10.1002/ jmv. 26625
19. Miesbach W. Pathological Role of Angiotensin II in Severe COVID-19. TH Open. 2020;4(2):e138-e44. doi:10.1055/s-00401713678

20. Gheblawi M, Wang K, Viveiros A, et al. Angiotensin-converting enzyme 2: SARS-CoV-2 receptor and regulator of the renin-angiotensin system: celebrating the 20th anniversary of the discovery of ACE2. Circ Res. 2020;126(10):1456-1474. doi:10.1161/CIRCRESAHA.120.317015

21. Zhang $\mathrm{P}$, Zhu L, Cai J, et al. Association of inpatient use of angiotensin-converting enzyme inhibitors and angiotensin II receptor blockers with mortality among patients with hypertension hospitalized with COVID-19. Circ Res. 2020;126(12):1671-1681. doi:10.1161/CIRCRESAHA.120.317134

22. Nishiga M, Wang DW, Han Y, Lewis DB, Wu JC. COVID-19 and cardiovascular disease: from basic mechanisms to clinical perspectives. Nat Rev Cardiol. 2020;17(9):543-558. doi:10.1038/ s41569-020-0413-9

23. Li C, Chen Q, Wang J, et al. Clinical characteristics of chronic liver disease with coronavirus disease 2019 (COVID-19): a cohort study in Wuhan, China. Aging (Albany NY). 2020;12(16):15938-15945. doi:10.18632/aging.103632

24. Moon AM, Webb GJ, Aloman C, et al. High mortality rates for SARS-CoV-2 infection in patients with pre-existing chronic liver disease and cirrhosis: preliminary results from an international registry. $J$ Hepatol. 2020;73(3):705-708. doi:10.1016/j. jhep.2020.05.013

25. Saini KS, Tagliamento M, Lambertini M, et al. Mortality in patients with cancer and coronavirus disease 2019: a systematic review and pooled analysis of 52 studies. Eur J Cancer. 2020;139:43-50. doi:10.1016/j.ejca.2020.08.011

26. Porfidia A, Valeriani E, Pola R, Porreca E, Rutjes AWS, Di Nisio M. Venous thromboembolism in patients with COVID-19: systematic review and meta-analysis. Thromb Res. 2020;196:67-74. doi:10.1016/j.thromres.2020.08.020

27. Barnkob MB, Pottegård A, Støvring H, et al. Reduced prevalence of SARS-CoV-2 infection in ABO blood group O. Blood Adv. 2020;4 (20):4990-4993. doi:10.1182/bloodadvances.2020002657

28. Zietz M, Zucker J, Tatonetti NP. Associations between blood type and COVID-19 infection, intubation, and death. Nat Commun. 2020;11(1):5761. doi:10.1038/s41467-020-19623-X

29. Bhattacharjee S, Banerjee M, Pal R. ABO blood groups and severe outcomes in COVID-19: a meta-analysis. Postgrad Med J. 2020; postgradmedj-2020-139248. doi:10.1136/postgradmedj-2020-139248

30. Alqahtani JS, Oyelade T, Aldhahir AM, et al. Prevalence, severity and mortality associated with COPD and smoking in patients with COVID-19: a rapid systematic review and meta-analysis. PLoS One. 2020;15(5):e0233147-e. doi:10.1371/journal.pone.0233147

31. Sin DD, Anthonisen NR, Soriano JB, Agusti AG. Mortality in COPD: role of comorbidities. Eur Respir J. 2006;28(6):1245-1257. doi:10.1183/09031936.00133805

32. Matsumoto K, Saito H. Does asthma affect morbidity or severity of COVID-19? J Allergy Clin Immunol. 2020;146(1):55-57. doi:10.1016/j.jaci.2020.05.017

33. Jackson DJ, Busse WW, Bacharier LB, et al. Association of respiratory allergy, asthma, and expression of the SARS-CoV-2 receptor ACE2. $J$ Allergy Clin Immunol. 2020;146(1):203-206.e3. doi:10.1016/j.jaci.2020.04.009

34. Chu Y, Yang J, Shi J, Zhang P, Wang X. Obesity is associated with increased severity of disease in COVID-19 pneumonia: a systematic review and meta-analysis. Eur J Med Res. 2020;25(1):64. doi:10.1186/s40001-020-00464-9 


\section{Publish your work in this journal}

The Journal of Multidisciplinary Healthcare is an international, peerreviewed open-access journal that aims to represent and publish research in healthcare areas delivered by practitioners of different disciplines. This includes studies and reviews conducted by multidisciplinary teams as well as research which evaluates the results or conduct of such teams or healthcare processes in general. The journal covers a very wide range of areas and welcomes submissions from practitioners at all levels, from all over the world. The manuscript management system is completely online and includes a very quick and fair peer-review system. Visit http://www.dovepress.com/testimonials. php to read real quotes from published authors. 